\title{
Layout validation and re-configuration in Plug\&Produce systems
}

Pedro Neves, Luis Ribeiro, Joao Dias-Ferreira, Mauro Onori and Jose Barata Oliveira

\author{
Journal Article
}

\section{Tweet}

N.B.: When citing this work, cite the original article.

Original Publication:

Pedro Neves, Luis Ribeiro, Joao Dias-Ferreira, Mauro Onori and Jose Barata Oliveira, Layout validation and re-configuration in Plug\&amp;amp;Produce systems, Assembly Automation, 2016. 36(4), pp.412-428.

http://dx.doi.org/10.1108/AA-12-2015-119

Copyright: Emerald

http://www.emeraldinsight.com/

Postprint available at: Linköping University Electronic Press

http://urn.kb.se/resolve?urn=urn:nbn:se:liu:diva-132560

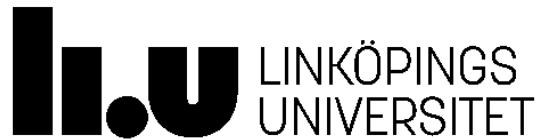


Title: Layout validation and reconfiguration in Plug\&Produce Systems

\section{Abstract}

Purpose - This paper aims to provide a method and decision support tool to enhance swift reconfiguration of Plug\&Produce systems in the presence of continuously changing production orders.

Design/methodology/approach - The paper reviews different production scenarios and system design and configuration methods and more particularly specify the need of decision support tools for Plug\&Produce systems that integrate configuration and planning activities. This problem is then addressed by proposing a method that helps reducing the solution space of the reconfiguration problem and allows the timely selection of the most promising reconfiguration alternative.

Findings - The proposed method was found to be helpful in reducing the reconfiguration alternatives that need to be considered and in selecting the most promising one for different orders. The advantages and limitations of this method are identified, and an illustrative test-case of the approach is presented corroborating the method applicability in the absence of large queues in the system.

Originality/value - This paper addresses a less explored domain within the Plug\&Produce systems research field, which is the system reconfiguration. It is proposed a method to support system validation and reconfiguration jointly with an illustrative testcase. This represents an original contribution to Plug\&Produce research field and it can have impact in improving agility and decreasing the complexity of reconfiguration activities to cope with constantly changing production orders.

Keywords-Plug\&produce systems, System reconfiguration.

Paper type - Research Paper

\section{INTRODUCTION}

The design and operation of automated production systems includes a set of complex decisions involving several conflicting goals. Decisions regarding equipment, layout, material flow, productivity, investment cost, scheduling, etc. lead to an enormous range of solutions (the "solution space"). These problems are typically solved through operations research and computer science algorithms and methodologies, resulting in trade-off solutions that compromise certain objectives in order to follow the strategic goals of the enterprise. Despite the fact that some of these models have proven to be profitable and robust when plans are stable, one fact remains undoubtedly true: they do not deal well with unexpected events and unpredictable production scenarios (Babiceanu and Seker, 2015, Neves et al., 2013).

As the business paradigm shifts towards an increase of customization and personalization of products, companies must deal with increasingly complex scenarios: high product diversity, complex supply chains, low inventories, small batch orders and strict deadlines (Hu et al., 2011, Maffei, 2012). To cope with these scenarios, two aspects become vital: Agile shop-floors that can be promptly (re-)deployed and (re-)configured with minimum integration and programming efforts; and decision support tools that can help dealing with the complexity of systems and support accurate decision-making based on collected data regarding available systems and equipment, shop floor capacity, current orders, etc. (Onori and Barata, 2009).

Several modern production paradigms have been introduced aiming at offering responsive and cost effective solutions to tackle these scenarios. Examples of these are Bionic Manufacturing Systems (Ueda, 1992), Reconfigurable Manufacturing Systems (RMS) (Koren et al., 1999), Evolvable Production Systems (EPS) (Barata et al., 2007), Holonic Manufacturing Systems (Valckenaers et al., 1997) and Changeable Manufacturing Systems (Wiendahl et al., 2007). These paradigms are grounded on the concepts of modularity and heterogeneity, and target the encapsulation of functionality in each module and their seamless integration in production systems. These are key aspects to tackle interoperability between systems, reduce the integration and set-up times, and increase tolerance to failures and re-usability of legacy equipment. To accomplish their design goals, they display a shift from centralized to distributed control architectures in which each node of the system is capable of taking autonomous decisions and interacts with the other nodes in the network.

Recent trends in shop-floor control research include the development of plug\&produce modules with scalable, interoperable and self-organizing control systems in order to enhance factory responsiveness (Jovane et al., 2008). Agent technology has been particularly popular to address manufacturing systems with plug\&produce ability, reconfigurability, robustness and responsiveness (Leitão and Karnouskos, 2015, Leitão et al., 2015). Agent technology enables that all entities in the shop floor (e.g. product, carrier, machine, etc.) are intelligent and able to reason, interact and exchange data in order to take decisions dynamically according to their context (Shen et al., 2006). Furthermore, agents seek the empowerment of local autonomy by using their self-* properties (i.e. self-configuration, 
self-diagnosis, self-organization, etc.) to form loosely coupled networks of agents that exhibit collective behaviour to autonomously tackle dynamic complex scenarios (e.g. new entity on the system, failures, etc.) (Ribeiro et al., 2008). Plug\&Produce (P\&P) systems typically rely on the concept of intelligent product (Wong et al., 2002, McFarlane et al., 2013), allowing each product/part to be transported independently and manage its own production by requesting transport and process executions. This makes them suitable to handle aspects related to the dynamic plug and unplug of production modules and change of process plans and volumes on the fly with near-zero reprogramming efforts (Leitão et al., 2015).

Despite its merits and achievements, P\&P systems are still at an infant stage. Before being commercially deployed at industrial level, their performance, robustness, scalability and reconfiguration abilities need to be extensively assessed. Moreover they should not be understood as a general panacea for all industrial scenarios but for targeted applications where they provide a profitable solution to tackle unpredictable markets where the products and volumes change very often. The biomedical market is such an example, where the miniaturized assemblies, zero defects constraints, high number of variants and unpredictable volumes together with the time and cost to develop, deploy and validate the assembly system reduces the application of automatic solutions (Williams et al., 2006, Onori and Barata, 2009). In markets where the number of product types, variants and possible offered customizations is high so is the overall system complexity which hardens the design and development of optimized cost-effective systems that can accommodate new products and volumes (Barata, 2005).

Most research in P\&P systems has focused on the development of interoperable pluggable modules, the necessary reference architectures and standard interfaces to support plugability and the real-time control system that renders the system autonomous and reactive to dynamic changes. Less attention has been drawn to the design and configuration of P\&P systems and methodologies that can support the adaptation and reconfiguration of P\&P systems to accommodate constantly changing requirements. These are major issues to be addressed since P\&P systems compromise slightly their performance in order to be more reconfigurable and fault-tolerant. Although the discussion widely applies to almost all emerging production paradigms, the Evolvable Production Systems (EPS) paradigm is used as a case to bring context and clarity to the discussion, and provide the necessary architectural guidelines to implement the work.

This article will be organized as follows: section II will provide a more detailed research framing and problem definition as well as all the necessary supporting concepts; section III will provide the main theoretical foundations of the proposed framework and the decision support tool' requirements and implementation details; section IV will

present a test-case to illustrate the proposed framework; finally section $\mathrm{V}$ will provide a critical discussion on the presented contributions and future research.

\section{LITERATURE ANALYSIS AND SUPPORTING CONCEPTS}

\section{A) State of the Art}

The production system design and configuration process aims at generating the most suitable configuration for the production of one or more product variants according to the production strategy. The generated configurations depend on the products' plans and try to satisfy the production and performance requirements by defining a layout, selecting resources and assigning tasks to resources. The possible configurations include a massive number of possibilities that reflect different system layout structures, assignments and operational decisions.

When the number of products and parts grow, so does the size of the system and necessary stations. This induces more complexity in the system design and makes analysis of productivity and quality for each configuration more difficult. The research community has undertaken a paramount effort to develop methods that can decrease the design complexity of product flow and process layouts. Different approaches using deterministic models, queuing models, discrete event simulation, axiomatic design and heuristics have been investigated to generate and evaluate configurations (Groover, 2007).

Deterministic models are used to roughly estimate the production rate, capacity and utilization; however typically they do not allow the evaluation of queues and other dynamics so they usually overestimate the performance (Groover, 2007). These include mathematical programming techniques that optimize a specific algebraic objective function of specific decision variables (Chryssolouris, 2006). These exact methods converge to a single solution which is sometimes not feasible due to some unpractical condition not being considered in the model (Shiyas and Pillai, 2014). For this reason, the generation of alternative configurations that can be further scrutinized by the designer can potentially lead to a more effective decision-support tool. 
Queuing models are used to describe the dynamics not present in deterministic approaches but usually allow only general modelling of queues and simple system configurations (Papadopoulos and Heavey, 1996).

Discrete event simulation (DES) is used in later stages of design and it provides the most accurate method to model the system dynamics (Groover, 2007). Nevertheless, it would be unfeasible to test all possible configurations using simulation due to time constraints and for this reason it is usually used in later design stages. A limiting factor of traditional DES is the dynamic decision making present in P\&P systems. This could be potentially tackled by using agent-based simulation where each agent in the shop floor is mirrored into a simulation environment to predict the dynamic decision take in different steps of production. A comprehensive review of DES publications regarding system design and operation can be found in (Negahban and Smith, 2014).

Axiomatic Design (AD) provides a formal design methodology that uses matrix methods to analyse the transformation of customer needs into functional requirements, design parameters, and process variables. An example is found in (Babic, 1999) where the use of AD provides the conceptual frame and methodology for FMS design and the criteria for its evaluation. Other interesting work using AD can be found in (Farid, 2015) proposing a formulation to measure the static resilience of a system and in (Farid and Ribeiro, 2014) where a multi-agent system reference architecture for RMS is quantitatively formalized.

Heuristics are usually applied to limit the solution space and achieve a time efficient solution. Classical exact solution methods (e.g. enumerative, branch and bound, linear programming, etc.) allow finding optimal solutions; however they can be extremely time-consuming when solving real-world problems (e.g. large-dimension, hardly constrained, etc.). Search methods find solutions by exploring paths in the solution space and the inclusion of a heuristic will dictate how the exploration will be performed. A strong heuristic can help to find suitable solutions in shorter time, but it can also fail if the best solutions are in the states it chooses not to try. Given the NP-complete nature of design problems (e.g. cell formation) heuristics have been widely used and examples can be found in (Renzi et al., 2014).

Due to the growing of the solution space (caused by the increase of workstations, product types, material flow, etc.) methods based on graph theory and complex networks are emerging to provide a structural analysis of the systems and detect potential bottlenecks or unbalanced workload distribution (Blunck et al., 2014, Becker et al., 2013, Becker et al., 2014). The traditional industrial view is critical towards these approaches mainly due to its level of abstraction which hardens the inclusion of important mechatronic and operational constraints (Becker et al., 2014). However they can provide an adequate tool to do preliminary system analysis and contribute to the reduction of the solution space and consequently reduce the number of morose simulations.

The current manufacturing context is characterized by mixed-model production with many different variants and fluctuating volumes. To deal with this variety and enable delayed product differentiation, the system layouts are often complex with asymmetrically branched configurations. Several algorithms have been proposed to try to automatically generate different configurations such as (Webbink and Hu, 2005, Michalos et al., 2012) however they cannot be applied to this work since they are focused on creating new layouts rather than re-configuring systems on the context of P\&P. Furthermore, in the case of (Webbink and Hu, 2005) another limiting factor is the assumption that all machines have the same functionality.

Recent work also includes the structural analysis of layouts and assessment of complexity. An example is found in (ElMaraghy et al., 2014) where the structural complexity of system layouts are assessed based on metrics that evaluate the number of connections, alternative paths, cycles, decision points and redundancy. Despite the usefulness of this work in assessing the structural complexity of a layout, there is no link between the product mix (types and volumes) to be produced, alternative layouts and the resultant material flow in the system and these aspects are fundamental for the work hereby presented.

\section{B) Problem Definition}

Traditional automation has been historically focused on the development of cost efficient mass-production systems for high volumes of standardized products. As the production strategy shifted towards mass-customization (Pine, 1993) the planning and implementation of automatic systems has become more complex. The continuous shortening of product volumes and life-cycles and the increment of product variants is increasing this complexity, to the point that automation of production is not cost-effective in some cases. The re-usability and swift re-configurability of an automated system is then of critical importance to attain cost-effective automation under these highly dynamic scenarios.

Traditionally, production systems are designed and configured according to forecasted conditions, and those activities are followed by planning and scheduling activities to optimise its operation. The planning and configuration 
activities in P\&P systems will necessarily be tightly coupled and performed periodically to effectively cope with new product variants, rush orders and volume fluctuations. Since these activities will overlap considerably, new methods and decision support tools are required to support the adaptation of P\&P systems to unforeseen production scenarios.

During their lifecycle, production systems can undergo physical (e.g. layout change, add/remove machines), logical (e.g. re-routing, re-planning, re-scheduling and re-programming) and parametric adaptations (e.g. change machine settings) (ElMaraghy, 2005). This is no exception for P\&P systems; nevertheless they are typically designed to handle logical and parametric updates autonomously. The physical adaptations are also targeted to be handled autonomously by the control system; however the changes of layout structure imply a re-engineering effort while the plug and unplug of machines can be done on the fly.

In the EPS context, P\&P is facilitated by common standard interfaces (mechanical, electrical and logical) and by a management system that runs in the background and keeps track of which resources are plugged in specific interfaces (Onori et al., 2012). These standard interfaces are denominated as docking points (DPs) and are connected by some kind of transport system (i.e. conveyors or AGVs). Changing the positions and connections of the available DPs (layout reconfiguration) implies re-engineering costs and associated development, test and deployment time. For this reason it is desirable that re-engineering is avoided and P\&P systems can be reconfigured quickly through the plugging/ unplugging of modules to timely follow market demands. In this sense, the performance of the system will be vastly dictated by the self-organization embedded in the system, the existent system layout (i.e. DPs positions and connections) and how the modules are distributed in the system (Neves et al., 2014a).

Upon new production requirements, the $\mathrm{P} \& \mathrm{P}$ ability can be exploited by devising different configurations (distribution of modules in the system) that may provide optimizations or enable the production of new products. P\&P systems support structure and functionality transformations through plug/unplug of modules, and dynamic production and fault-tolerance through self-organization. This will naturally increase complexity in design, operation and exact performance predictability, and therefore sets the need for the definition of reconfiguration methods and decision supporting tools that can help system designers and production managers to decide which layouts and configurations could accommodate specific production requirements (i.e. different product plans and volumes).

The main focus of this work is to validate existing P\&P layouts and to reduce the configuration alternatives presented to the user. In the context of this work, configuration means exclusively the distribution of the modules in a fixed P\&P system layout structure and the goal is to verify if there is at least one reconfiguration that satisfies the new production requirements. If so, the reconfiguration alternatives should be assessed in a timely manner. In this sense, this article's main contribution lies in the proposal of a reconfiguration method and metrics that support the timely generation and assessment of reconfiguration alternatives by employing solution space reduction. The proposed metrics assess the suitability of reconfiguration alternatives to cope with the production flows resulting from dynamic orders. To achieve this, the proposed approach abstracts system configurations and production plans as graphs and uses graph theory concepts to conclude about the potential performance of different reconfiguration alternatives.

\section{C) Relation to graph theory and system formulation}

Graph theory provides powerful concepts that can facilitate the evaluation of P\&P systems. Informally a graph is a set of nodes joined by a set of arrows. Representing a problem as a graph can provide a different point of view, making the problem simpler and possibly solvable. Graph structures can be used to isolate interesting or important sections of a graph while structural metrics provide a measurement of a structural property of a graph (globally or locally). The identification of specific structures or relations in a graph can then allow solving the represented problem.

Graphs have been used to provide structural descriptions of complex systems and their interactions. They have been successfully employed to model self-organizing systems (bio-chemical reactions, social interactions, etc.) and also pattern recognition and computer vision analysis. From the assembly point of view graphs have also been employed especially in the assembly plan representation. They proved to be useful to model modular products, product families and assembly plans with and/or precedence constraints (Hu et al., 2011, Yu and Li, 2006). Graphs can also be employed to represent assembly system layouts. In P\&P systems the nodes can represent the DPs where process-oriented workstations/modules are deployed, while the edges can represent the connection between them (conveyor system or AGV-based transport system).

Formally a graph $\mathrm{G}=(\mathrm{V}, \mathrm{E})$ is a representation of a set of objects (set of vertices $\mathrm{V}$ ) where some pairs of objects are connected by links (set of edges $\mathrm{E}$ ). If the edges have a specific orientation then the graph is directed while if they do 
not the graph is undirected. A graph can also be a weighted graph if the edges have weights or unweighted if they do not. A weighted, directed graph $\mathrm{G}=(\mathrm{V}, \mathrm{E})$ has a weight function $\mathrm{w}: \mathrm{E} \rightarrow \mathrm{R}$. The weight of a path $\mathrm{p}=<\mathrm{v}_{0}, \mathrm{v}_{1}, \ldots, \mathrm{v}_{\mathrm{k}}>$ is the sum of the weights of its constituent edges, as depicted in Equation 1. A shortest path from vertex $u$ to vertex $v$ is then defined as any path $\mathrm{p}$ with weight $\mathrm{w}(\mathrm{p})=\delta(\mathrm{u}, \mathrm{v})$.

Equation 1 - Weight function and shortest path in directed graphs

$$
w(p)=\sum_{i=1}^{k} w\left(v_{i-1}, v_{i}\right)
$$

The shortest path weight from $\mathrm{u}$ to $\mathrm{v}$ is:

$$
\delta(u, v)=\left\{\begin{array}{l}
\min \{w(p)\} \\
\infty
\end{array} \text { if there is a path } p \text { from u to } v,\right.
$$

A graph $F$ is a subgraph of a graph $G(F \subseteq G)$ if:

- $\quad V(F) \subseteq V(G)$, i.e. the vertices set of $F$ is contained in the vertices set of $G$ and;

- $\quad E(F) \subseteq E(G)$, i.e. the edges set of $F$ is contained in the edges set of $G$.

These and other graph theory definitions can be found in (Gross and Yellen, 2005).

Considering the system formulation presented before, a manufacturing system is envisioned as a directed, weighted graph whereby the DPs are the nodes and each edge denotes one path connecting two DPs (see Figure 1). The edge direction is important since it gives information regarding the flow in the conveyors (c1, c2, etc. in Figure 1) or AGVs and this is fundamental to know if ordered processes can be executed. The edge weight is also important since it gives measurable information regarding the transport system (e.g. weight can represent distance, speed, travel time, etc.). Edges' weight can also represent the flow of products in the system or any other information that is considered relevant. The routers of the system (r1, r2 in Figure 1) do not need to be represented in the graph since they are merely mechatronic details.

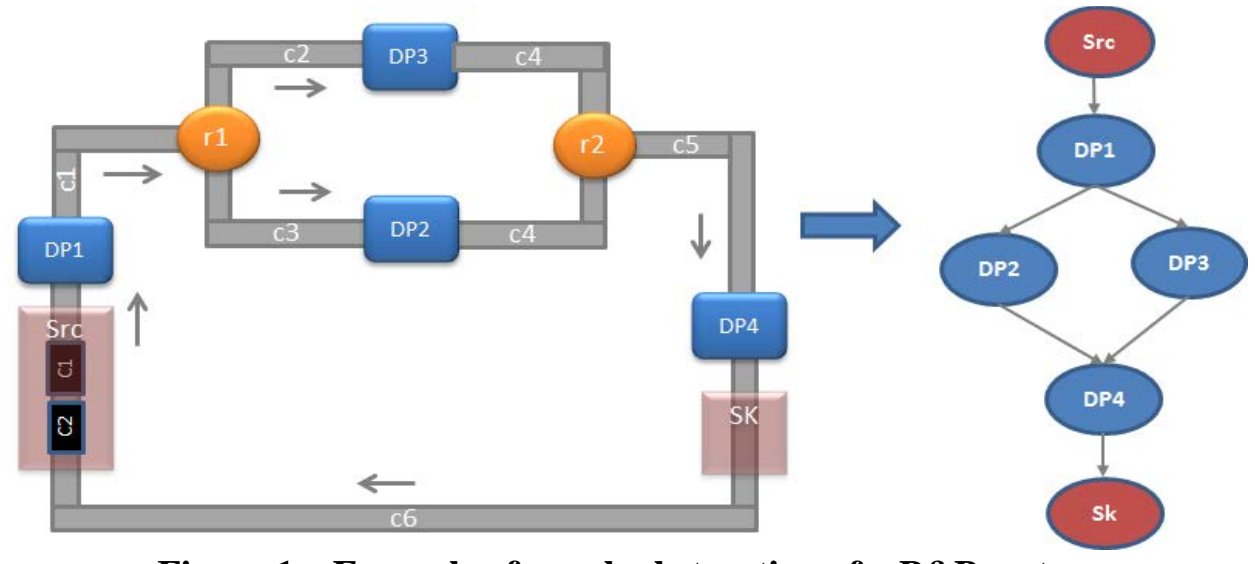

Figure 1 - Example of graph abstraction of a P\&P system

The system formulation includes a Source point (Src) at which the carriers enter the system and a Sink point (Sk) where the carriers leave the system. This is still considered valid for cases in which the system has a closed loop (as seen in Figure 1) since the configuration alternatives that force the carriers to do loops in the system should be avoided to reduce queues and unnecessary travel/waiting times. Currently this work considers exclusively systems that have only one source and one sink point. Regarding the product plans, the sequence of production processes can be represented through a directed graph where the nodes are the processes that need to be executed and the edges denote the order of execution. It is assumed that the graphs representing both the system and the production processes have no loops. Both graphs are directed to capture the direction of the possible material flow in the system, in the first case, and the order of process execution in the second case. The description on how this modelling and techniques come together for the purpose of this work is detailed next. 


\section{Proposed APPROACH FOR RECONFIGURATION GENERATION AND ASSESSMENT}

\section{A) Reconfiguration activities in EPS methodology}

In P\&P systems, changing requirements can be tackled by changing the structural relations and global behaviour of the system through plug and unplug of autonomous self-contained modules in the available DPs. It is possible to change modules/processes in the system, scale or optimize it with near-zero reprogramming and re-integration time. This entails a competitive edge to deal with turbulent markets since systems can be swiftly reconfigured to better match current orders and fulfil all necessary functional (e.g. processes) and operational (e.g. throughput time) requirements.

Orders can be accepted or rejected by analysing the current shop floor status (production times, WIP, available modules, available systems, etc.) and cost/performance evaluation of the needed reconfiguration. The accepted orders can be grouped according to delivery date and priority and assigned to specific P\&P systems. The P\&P system can then be periodically, reconfigured to better match the orders that will be produced in the following period. This means that the modules can be plugged optimally according to current production requirements by performing a reconfiguration analysis that considers factors such as mechatronic constraints, material flow, station balancing, etc. Naturally, even in self-organizing production, the system response will only be as good as its physical limitations. For example, despite using the best routing algorithms, if the distribution of modules in the system does not group processes to minimize the necessary travel and waiting times this will result on low performance. Since the concept of P\&P and self-organizing production lays on the reusability and reconfiguration of systems it is then important to not only guarantee production upon new production requirements but also to try to optimize the system through reconfiguration.

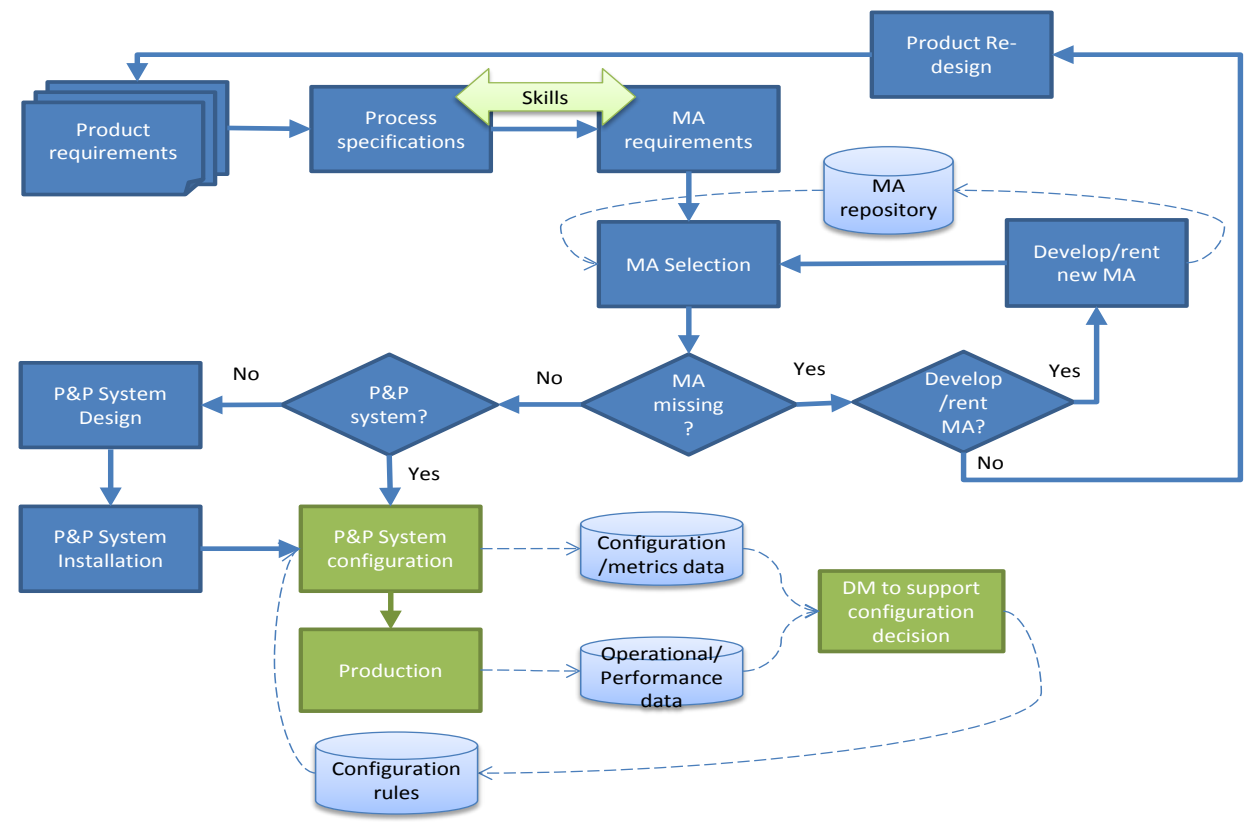

Figure 2 - Area of research highlighted

In Figure 2 the area of research is highlighted in green, extending the EPS methodology presented in (Maffei and Onori, 2011). When new product requirements arise they are translated into process specifications and subsequently into module requirements. According to these requirements and the module repository, the modules can be selected. If some modules are not available, the company has several options. It can choose to rent or develop that module, redesign the product or decide to not accept the order if it is not economically viable. If all indispensable modules are available, the company can assess the suitability (in terms of layout, material handling, current orders, etc.) of the available P\&P systems to produce the required products. If no system is available, an evaluation is performed to realise if a new P\&P system design and consequent installation should proceed or if the business opportunity is not considered profitable. This article does not include any methodology or guidelines to design optimal P\&P system layout structures that enhance its reconfiguration since this is considered future research. 


\section{B) Method for reconfiguration generation and assessment}

Considering the system formulation in the previous section, production modules can be plugged into any DP. Assuming there is a set of available modules that offer all the different processes necessary to produce the current orders then a P\&P system planner must decide how to distribute the modules in the DPs to guarantee that all products can be produced and that the best level of performance (without re-engineering the system) can be achieved. The proposed method to perform the P\&P system configuration according to the collected orders is depicted in Figure 3. The first two steps consist in the definition of the system layout features and the process plans as weighted directed graphs, as well as the definition of all available production modules.

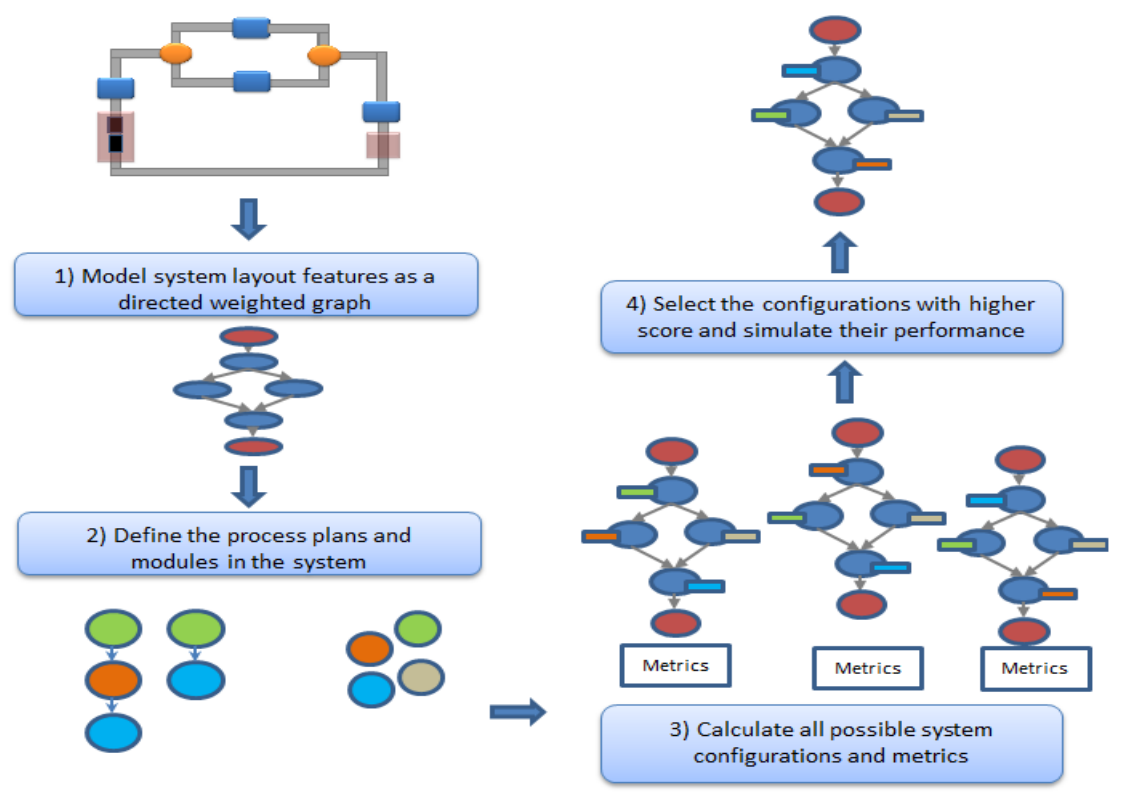

Figure 3 - Proposed method to perform P\&P system configuration

In step 3, all the possible system configurations are generated and assessed. Assuming $\mathrm{N}$ modules will be deployed in N DPs the total number of possible configurations is $\mathrm{N}$ factorial (N!). However since both the system's and the process's graphs are directed, many of the theoretical possible configurations are not feasible since they would not support all the production steps. If one product requires the execution of processes $\{A, B, C\}$ then the system must necessarily have a valid path that connects process A to B and B to C (even if in an indirect way). During step 3 the graph that represents the system layout is populated with the modules in all possible combinations generating $\mathrm{N}$ ! graphs that represent all possible configurations (see Figure 4). Each configuration graph is then matched against the process plans' graphs (see Figure 5). If all necessary paths to execute the process plans are found the configuration is validated, otherwise it is discarded. As depicted in Figure 5, the process plan 1 and 2 (PP1 and PP2) can be executed in the tested system configuration, even if PP2 does not have direct paths for all the processes (PP2 graph is not a subgraph of the system configuration graph). However, note that this particular configuration is not valid to execute the process plan 3 (PP3) since in that configuration there is no path linking a screw process to a glue process.

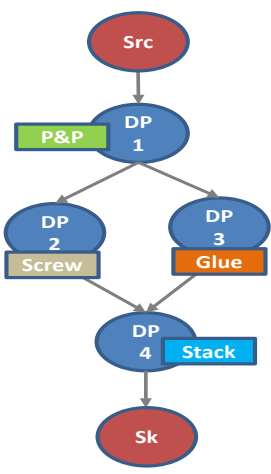

Configuration 1

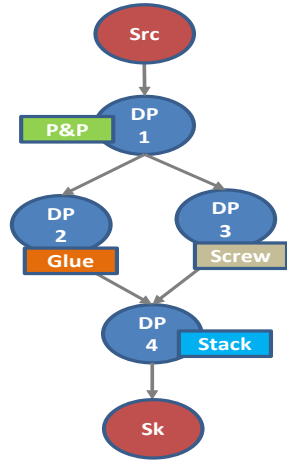

Configuration 2

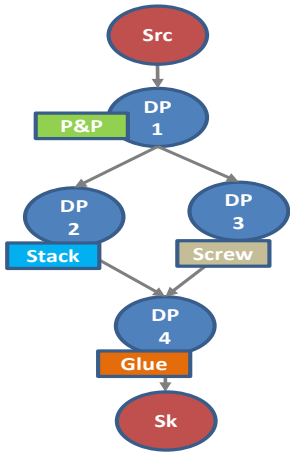

-. Configuration $\mathrm{N}$

Figure 4 - Generation of all reconfiguration alternatives 
This matching process will potentially largely reduce the number of possible configurations that need to be subsequently analysed. The authors do acknowledge that the factorial nature of this computation is time consuming and computationally intensive, however the generated configurations would indeed be in the set of options that would have to be considered by system designers or production managers.

The thorough examination and simulation of all candidate solutions would be prohibitively time consuming and for this reason, metrics that scrutinize the structural similarity between the selected configurations and the process plans as well as evaluate the potential travel and waiting times are presented in the next section. In step 4, the proposed metrics are employed to rate all candidate solutions and support the reconfiguration decision. The reconfigurations with higher score (i.e. maximize the performance goals) can be subsequently analysed through simulation.

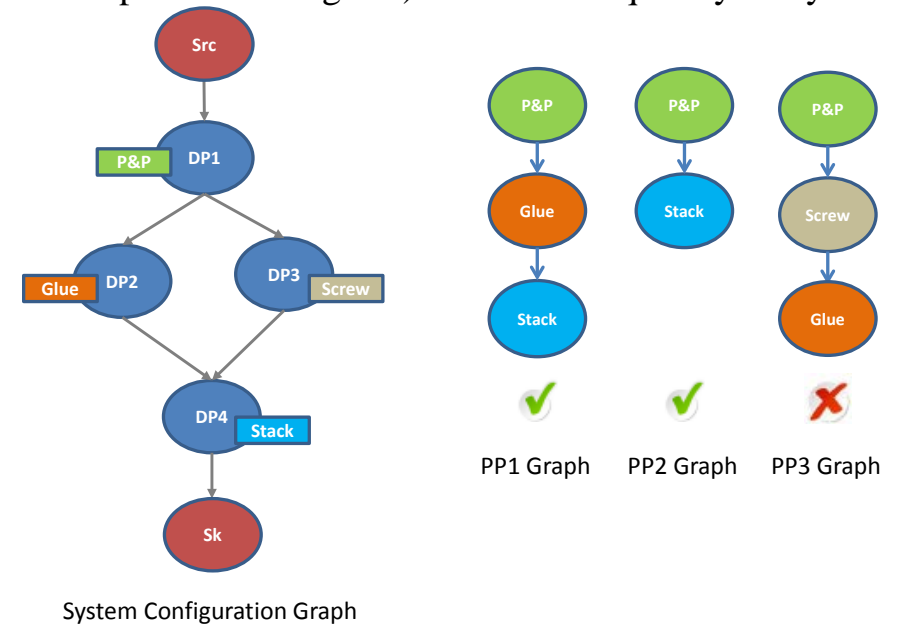

Figure 5 - Matching between system reconfigurations and process plans

C) Performance goals

In order to reduce the response time to customer orders (throughput time ) a system can be optimized in different ways : Reduce setup times, processing times, transport times and waiting times (Johnson, 2003).

Setup times can be reduced, for example, by using dedicated equipment. The P\&P modules are considered to be dedicated; hence the presented work will not cover this aspect.

The processing time can be reduced by reducing time per operation (which can also be achieved by the use of dedicated equipment). It can also be reduced through reducing scrap or rework by using one piece flow which is also considered in this research since products/parts travel independently and orchestrate their own production.

The waiting times can be reduced mainly by increasing resource access, reduce process variability by using dedicated equipment and reduce the number of queues. In the context of P\&P systems this will include the removal of non-adding value stations throughout the production path and reducing the queuing time by balancing the load in stations and conveyors.

The transport time can be reduced by decreasing the time per move and the number of moves. This can be achieved by grouping equipment performing sequential operations, improve layout and/or increase station capacity.

These main factors influencing setup, processing, transport and waiting times are implicit in this research but the main focus of this approach will be on reducing transport time and the waiting time by selecting the best reconfiguration for the current P\&P system layout based on the metrics proposed in the next section.

\section{D) Reconfiguration assessment}

In typical self-organizing production there is no pre-defined task-resource allocation since this process is done in runtime. Hence to evaluate reconfiguration alternatives this assignment has to be estimated. In this work the routing decisions are based on a probability function inverse to the route cost, where the route cost is calculated based on the estimated travel time (without queues). Routes with less cost have therefore more probability to be selected, which provides a realistic approximation of the distribution of products in the stations as given by the self-organizing product routings. For a set of $n$ possible paths to execute a process, each path $p_{w}$ has a cost $c_{w}$ and probability $P\left(c_{w}\right)$ of being selected, as depicted in the Equation 2. An example of calculation of the path routing probability function is depicted in Figure 6, where it is possible to observe the influence of the route cost in the estimation of resource assignment. In the figure is depicted the route $\operatorname{cost} c_{w}$ between each pair of DPs. 


$$
\begin{aligned}
& \text { Equation } 2 \text { - Path ro } \\
& P\left(c_{w}\right)=\frac{\frac{1}{c_{w}} * 100}{\sum_{w=1}^{n} \frac{1}{c_{w}}}
\end{aligned}
$$

The proposed metrics do not consider queues and other dynamics such as buffers but they do capture several physical system limitations present in the configuration. Moreover they are designed separately to deliberately allow the system user to understand the potential problems with specific configurations rather than optimizing a unique cost function. This leads to a more user-friendly, readable result and enables future research on the application of machine learning to unveil the relation between the configuration metrics and the system performance.
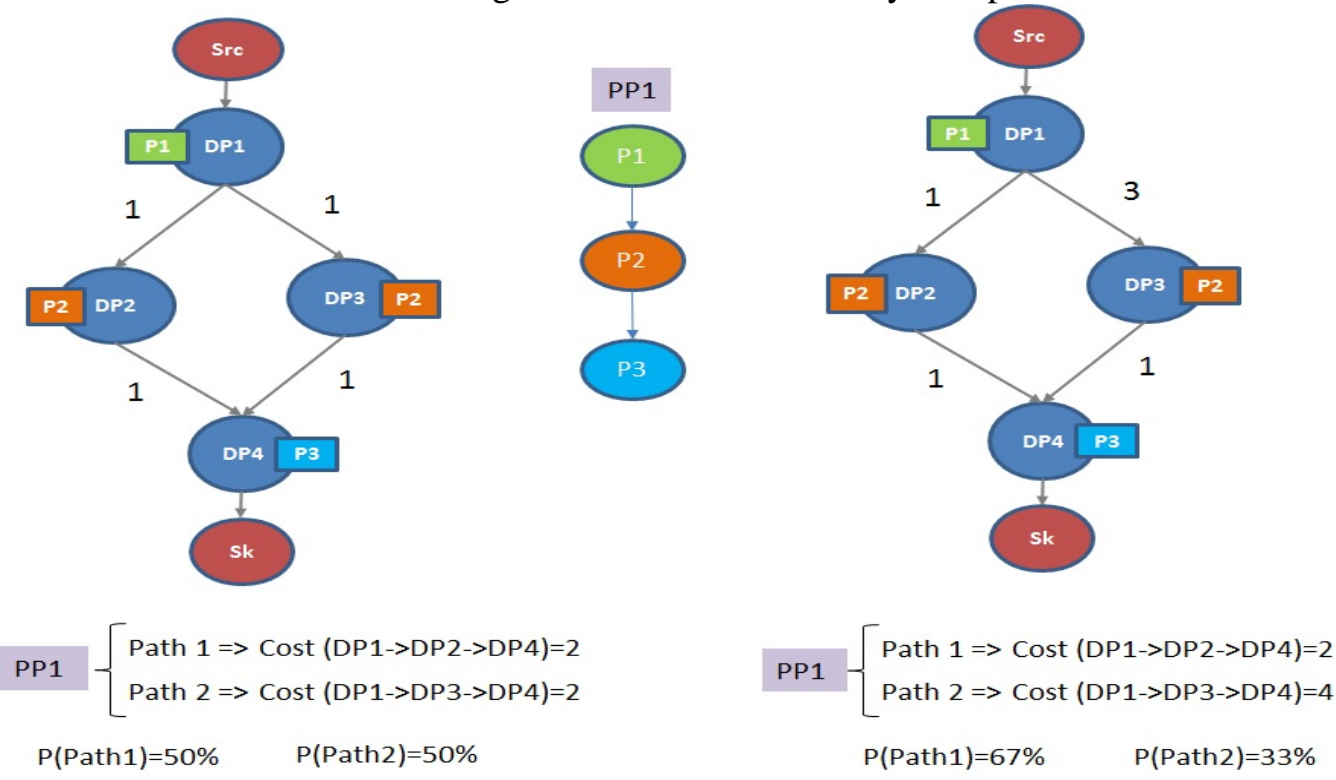

Figure 6 - Path routing probability example

\section{Reduce move time metrics}

The proposed metrics capture the suitability of the system to execute the defined process plans. The physical constraints on the system configuration are measured by the move speed and distance necessary to implement the process plans, as well as the grouping of sequential operations to avoid travelling through non-value adding stations.

\section{Process System Affinity Index (PSAI)}

PSAI evaluates how strictly the required process plans adhere to the structure of the evaluated system configuration $G_{\text {sys }}$ by measuring if all the sequential processes required for the execution of a specific process plan $G_{i}$ are grouped optimally, i.e. they can be routed without travelling through non-value adding stations. As depicted in Equation 3, this is achieved through subgraph matching between every production process plan graph $G_{i}$ and the system configuration graph $G_{\text {sys }}$ and by counting the number of vertex hops between the source point and process 1 $\left(h_{\text {Src }}\right)$ of $G_{i}$ and those between the last process in $G_{i}$ and the sink point $\left(h_{\text {sk }}\right)$. The hop count gives this metric a global context and ensures the minimization of travel through/to non-value adding stations.

\section{Equation 3 - PSAI metric equation}

PSAI $=\sum_{i=1}^{n} \frac{P_{i}}{P_{t}} f\left(G_{i}\right)$

where $f\left(G_{i}\right)=\left\{\begin{array}{cl}\frac{2}{h_{S r c}+h_{S k}} & \text { if } G_{i} \subseteq G_{\text {sys }} \\ 0 & \text { otherwise }\end{array}\right.$

In the ideal system configuration, all production process plans are subgraphs of the system configuration $\mathrm{G}_{\text {sys }}$, the station that executes the first process in the process plan is an outbound connection of the source point, and the sink 
point is an outbound connection of the station that executes the last process. In this case the metric will be equal to 1 (maximum value). In this measure the volume of each process plan is also considered. It is represented by the ratio of $\mathrm{Pi} / \mathrm{Pt}$ where $\mathrm{Pi}$ is the number of products of process plan $\mathrm{i}$ and $\mathrm{Pt}$ is the total number of products in the current order. This is important since it represents the affinity with the volumes of each process plan contributing to a more accurate measure. An example is provided in Figure 7, where it is possible to observe the metric result with different process plans.
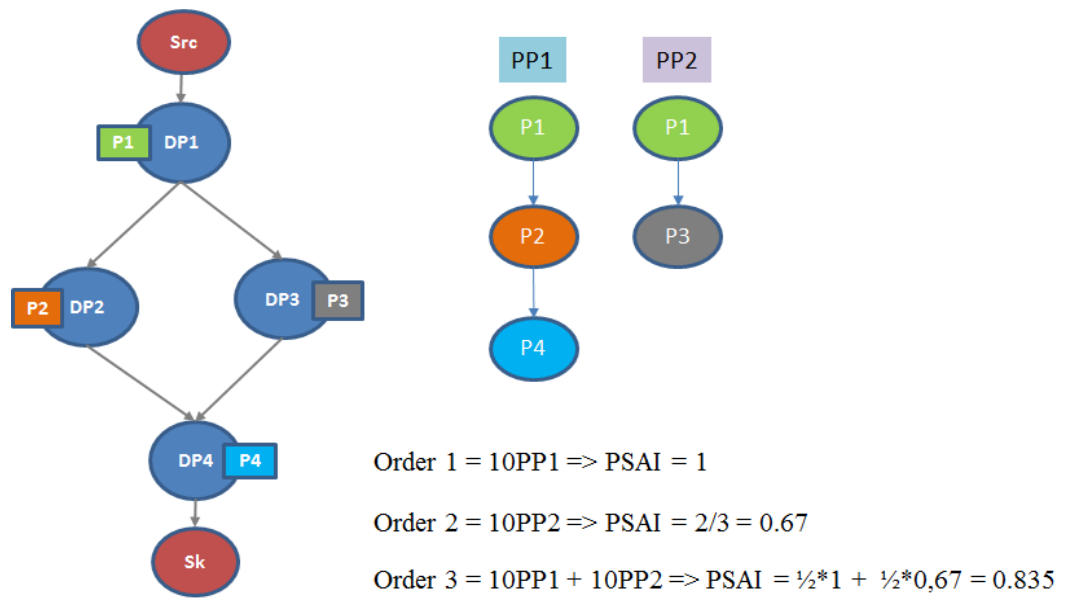

Figure 7 - Example of PSAI metric

\section{Move Time Index (MTI)}

The MTI is used to measure the move distance and speed. It considers the move cost for the $n$ process plans and weights them according to their volume $\left(\mathrm{P}_{\mathrm{i}}\right.$ is the number of products of process plan $\mathrm{i}$ and $\mathrm{P}_{\mathrm{t}}$ the total number of products in the current order). This guarantees that system configurations will be rated according to the volumes of products of different types. The equation to calculate MTI is depicted in Equation 4.

$\mathrm{Cp}_{\min }$ corresponds to the theoretical minimum result and it is achieved by multiplying the minimum number of paths necessary to travel to accomplish the specific process plan i (number of vertex hops hops ${ }_{\min }$ ) by the minimum cost for each path (minimum distance per path $\mathrm{D}_{\min }$ divided by maximum speed per path $\mathrm{S}_{\max }$ ). The minimum number of vertex hops corresponds to the number of processes to be executed plus one. For example if the process plan includes the execution of two processes the minimum vertex hops will be equal to 3 (source to process 1 , process 1 to process 2, process 2 to sink). This number of hops means that no non-value adding stations are crossed during the execution. $C p_{i}$ corresponds to the total transport time cost (distance $D_{j}$ divided by speed $S_{j}$ ) path by path for the $m$ paths included in the selected path.

\section{Equation 4 - MTI metric equation}

$$
\begin{aligned}
& \text { MTI }=\sum_{i=1}^{n} f\left(G_{i}\right) \\
& \text { where } f\left(G_{i}\right)=\frac{P_{i}}{P_{t}} \frac{C p_{\min }}{C p_{i}} \\
& \text { and } C p_{\text {min }}=\text { hops }_{\text {min }} x \frac{D_{\text {min }}}{S_{\text {max }}}, \quad C p_{i}=\sum_{j=1}^{m} \frac{D_{j}}{S_{j}}
\end{aligned}
$$

Considering the ratio of distance and speed as the weight of each edge/path, then it is clear that this corresponds to the ratio between the minimum possible weighted shortest path (in an ideal system graph) and the selected weighted path that exists in the tested configuration. This index will be at most 1 and that means that the system configuration has a transport cost equal to the theoretical minimum for all the process plans. An example is depicted in Figure 8, where it is possible to observe the influence of conveyor distance in the metric result. 

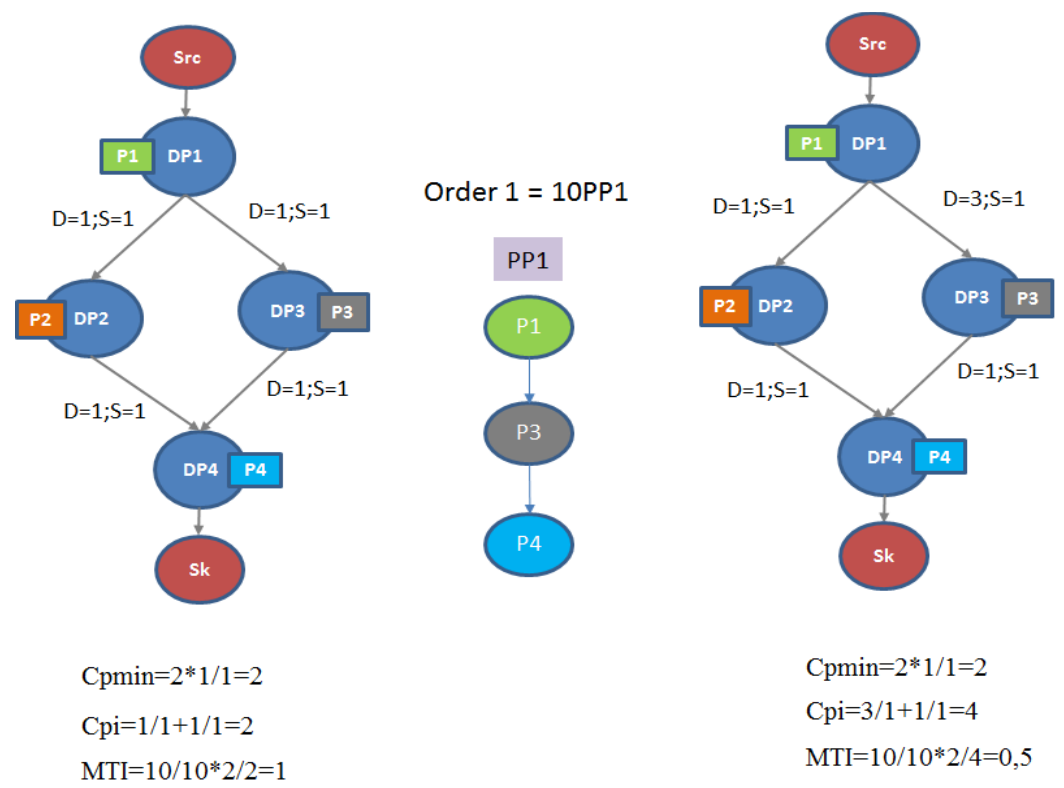

Figure 8 - Example of MTI metric

Reduce waiting time metrics

The accurate measure of the formation of queues and consequent bottlenecks and waiting times requires the knowledge of several parameters such as station workloads, cycle times, buffers, etc. However it is possible to have an estimation of the suitability of the reconfiguration in terms of waiting times by analysing the distribution of products in the transport system and stations and also by increasing resource access through different routing options.

\section{Path Distribution Index (PDI)}

Recurring to the estimated assignment based on the probabilistic function presented above, it is possible to estimate the distribution of products in the transport paths. Obviously the products will not be in the paths all at the same time (depends on which order the products enter the line, the process times, etc.) but a high unbalance of the load in the paths can be an indicator of queues in the system. This analysis is also useful to identify paths where no transport of products exists at all.

The Gini Index is used to relate the load in the paths and present the results in an index format, which measures the inequality among values of a frequency distribution and is typically used to present results on the income inequality distribution (Gastwirth, 1972). In this case the input will be the number of products allocated to each path rather than the income of an individual. The Gini index can be calculated from unordered size data calculating the mean of the difference between every possible pair of path loads x, divided by the mean size $\mu$ (Dixon et al., 1987), as depicted in Equation 5. A Gini index equal to 0 is an indicator of perfect equality among the path loads while a Gini index equal to 1 represents total inequality. In order to be coherent with the other metrics and to facilitate the readability of the reconfiguration scores the PDI metric will be presented as 1-Gini as depicted in Equation 6. It results then that perfect equality on path distribution will be reflected on a PDI score equal to 1.

\section{Equation 5 - Gini index as presented in (Dixon et al., 1987)}

$$
G=\frac{\sum_{i=1}^{n} \sum_{j=1}^{n}\left|x_{i}-x_{j}\right|}{2 n^{2} \mu}
$$

\section{Equation 6 - PDI metric equation}

$$
P D I=1-G
$$

\section{Workload distribution Index (WDI)}

This metric is analogous to the previous one but instead of considering the load in the paths it considers the load in the stations. The workload of each station is calculated by multiplying the number of products executing a process in that station with the time it takes to execute that process.

If the stations' workload is well balanced then the Gini index will be close to 0, otherwise it will be higher. A high index could mean the presence of bottlenecks due to uneven distribution of work. The authors do acknowledge the 
fact that some inequalities can be caused by the lack of redundancy of some processes or by the extensive use of specific processes in the process plans compared to other processes. Nevertheless since the goal is to compare different reconfigurations, this metric can potentially be useful to rank them. As with the previous metric the result will be presented as 1-Gini as depicted in Equation 7.

\section{Equation 7 - WDI metric equation}

$W D I=1-G$

An example where the PDI and WDI metrics are employed is presented in Figure 9, where it can be observed the number of products that, according to the previously calculated path routing probability function, request process and transport executions in the different stations and paths.
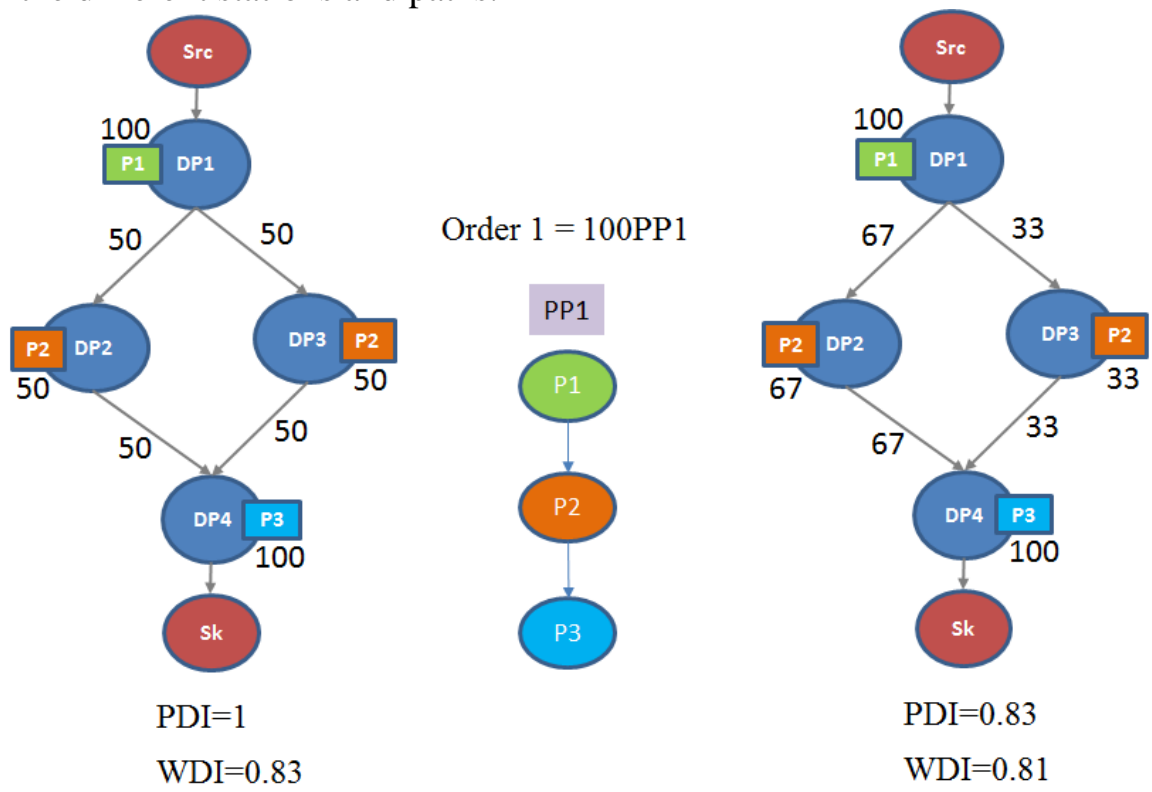

Figure 9 - Example of PDI and WDI metric

\section{Routing Flexibility Index (RFI)}

This metric captures the routing flexibility and resource accessibility (i.e. stations reachable or not) of the reconfiguration by calculating the total number of alternative full paths $n_{\text {paths }}$ that exist to execute each of the $n$ process plans (see Equation 8). For a full path to be considered valid it needs to allow the execution of all processes in the process plan and have a link with the source and sink points. The full paths are calculated by using a DepthFirst Search (DFS) algorithm to find all the DPs/production modules the product needs to visit in order to fulfil its process plan. This algorithm iteratively finds all paths for each process pair in the process plan, i.e. it finds all the paths from the first process in the process plan to the second one and then from the final point of the found paths it calculates the possible paths to the next process plans. In the end all possible path combinations are assembled resulting in the total alternative full paths $n_{\text {paths }}$.

\section{Equation 8 - RFI metric equation}

$$
R F I=1-\frac{1}{\sum_{i=1}^{n} \frac{P_{i}}{P_{t}} f\left(G_{i}\right)}
$$

where $f\left(G_{i}\right)=n_{\text {paths }}$

Similarly to the previous metrics, the volumes of each workflow are considered and, in which $\mathrm{P}_{\mathrm{i}}$ corresponds to the number of products of process plan $\mathrm{i}$ and $\mathrm{P}_{\mathrm{t}}$ is the total number of products in the current order. This metric score will have a maximum of one when several alternative paths exist to produce all workflows tested. The RFI metric equal to 0 means that only one path exists that guarantees the execution of each process plan. The bigger the score of this metric the more alternatives exist to execute the process plans and, therefore, the bigger is the routing flexibility and 
resource accessibility. An example is depicted in Figure 10, in which it is possible to observe different routing flexibility for two reconfigurations.

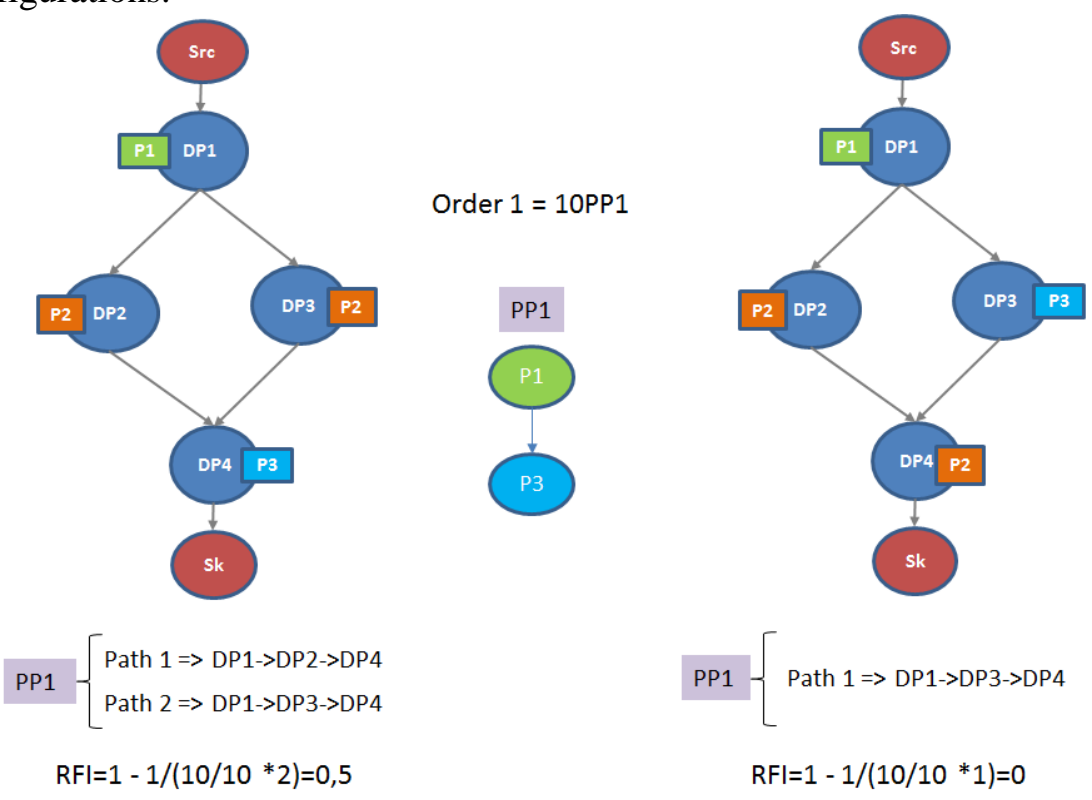

Figure 10 - Example of RFI metric

\section{Test-Case}

A) Supporting architecture and implementation details

In the current version a framework for testing the proposed method has been developed based on a simulation tool that simulates the operation of the self-organizing plug\&produce system with different layouts and configurations. This tool has been developed under the umbrella of the EPS paradigm and derived from the IADE architecture (Ribeiro et al., 2012). The simulation tool initially published in (Neves et al., 2014b) was extended under the scope of this article with the layout generator with exhaustive search of all combinatorial possibilities and rating.

In the present work, the tool allows the user to introduce the system layout (with DPs, distance of paths connecting different DPs, speed of paths, etc.), production modules (name of module, performed process and process time) and the production plans through a Graphical User Interface (GUI). It uses the Jung library (O'Madadhain et al., 2003) to support the visualization and analysis of the graphs and the Combinatorics lib 2.1 (Paukov, 2015) to support the generation of all candidate solutions. After the candidate reconfigurations are selected, according to the previously defined metrics, the user can proceed with the simulation of the selected candidate solutions as detailed in (Neves, Ribeiro et al. 2014).

The tool can be used in two different ways: exploratory manner, where the user introduces the layout and modules and all candidate solutions are generated and rated; and expert mode, where the user introduces the layout and modules but also defines the allocation of certain modules to certain DPs (due to constraints on size, buffers, material, etc.). In this case the space exploration will be reduced leading to fewer reconfigurations and faster results. In the extreme case, the user allocates all modules to the DPs and the metrics will be calculated only for that specific reconfiguration.

\section{B) Test-case Description}

To illustrate the proposed approach, metrics and infer its validity, a test-case is presented with a P\&P system with 8 DPs and a structure as depicted in Figure 11. For the sake of result readability and illustration of the approach the designed P\&P system could not be too large or complex. Nevertheless it provides enough complexity to render it interesting to study since it has several DPs and routing alternatives and therefore enables the generation of several alternative reconfigurations.

It is considered that all the conveyors (c1, c2, etc.) connecting the different DPs are 4 meter long and when routers (r1, r2, etc.) are necessary to merge/split conveyors these are included in the total distance. The system has asynchronous transport provided by 12 autonomous carriers (C1, C2, etc.) that move in single-direction. They travel on the conveyors at 1 meter/second and each carrier can transport only one product at a time. The number of carriers 
was dimensioned to avoid overloading the system and generate queues. The execution of a product starts when it is loaded into a carrier in the Source point (Src) and the loading operation takes 3 seconds. When the product execution finishes, the carrier is routed to the Sink point (Sk) to unload the product and the carrier becomes available again in the Source point. To simplify readability of results, no redundancy was used in the presented tests. It is therefore expected that the WDI metric will remain constant for all reconfiguration alternatives of a specific order.

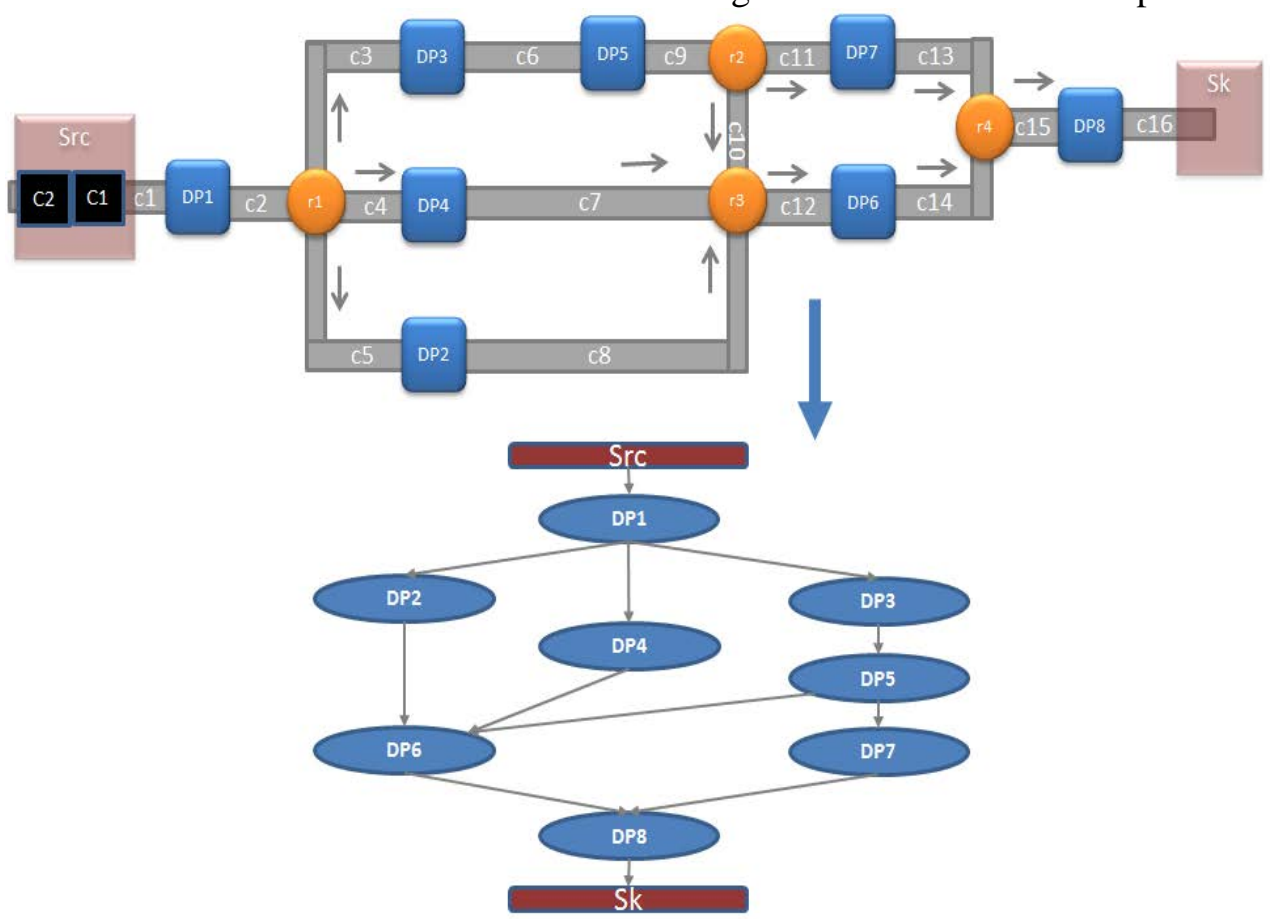

Figure 11 - P\&P system used in this test-case (at the top) and correspondent graph abstraction (at the bottom)

The presented test-case considered a sequence of 7 orders (see Table 1 and Figure 12) that require the execution of 5 different process plans (PP): $\mathrm{PP}_{1}=\left\{\mathrm{P}_{1}, \mathrm{P}_{2}, \mathrm{P}_{6}\right\}, \mathrm{PP}_{2}=\left\{\mathrm{P}_{3}, \mathrm{P}_{5}, \mathrm{P}_{7}\right\}, \mathrm{PP}_{3}=\left\{\mathrm{P}_{1}, \mathrm{P}_{6}, \mathrm{P}_{8}\right\}, \mathrm{PP}_{4}=\left\{\mathrm{P}_{1}, \mathrm{P}_{4}, \mathrm{P}_{6}\right\}$ and $\mathrm{PP}_{5}=\left\{\mathrm{P}_{5}, \mathrm{P}_{7}, \mathrm{P}_{8}\right\}$.

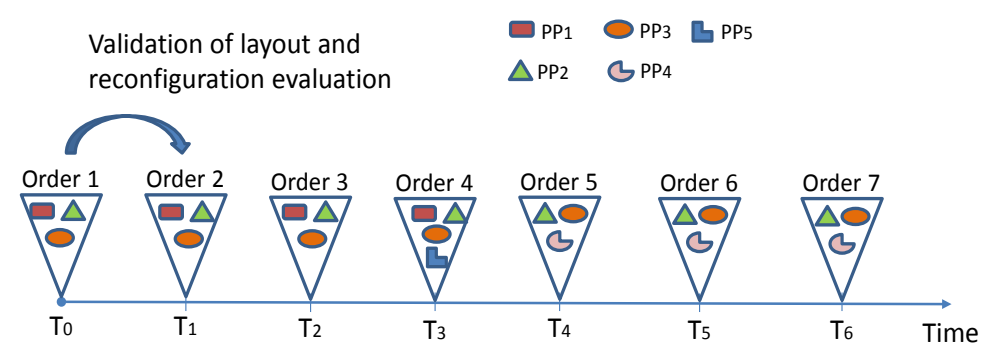

Figure 12 - Orders considered in the test-case

As depicted in Figure 12, upon the introduction of a new order there is an assessment of reconfiguration alternatives that can better match the order requirements. The considered orders were pre-defined and not randomized to ensure repeatability of the tests and results. The orders are, however, independent and the proposed approach evaluates one at a time without considering possible future orders. The available modules and respective process name and processing time are depicted in Table 2.

Table 1 - Orders in the test-case with the number of products of each process plan (PP) and total amount

\begin{tabular}{|c|c|c|c|c|c|c|}
\hline Order & $\mathrm{PP}_{1}$ & $\mathrm{PP}_{2}$ & $\mathrm{PP}_{3}$ & $\mathrm{PP}_{4}$ & $\mathrm{PP}_{5}$ & Total \\
\hline 1 & 100 & 10 & 10 & 0 & 0 & 120 \\
\hline 2 & 10 & 100 & 10 & 0 & 0 & 120 \\
\hline 3 & 10 & 10 & 100 & 0 & 0 & 120 \\
\hline 4 & 10 & 10 & 10 & 0 & 150 & 180 \\
\hline
\end{tabular}




\begin{tabular}{|c|c|c|c|c|c|c|}
\hline 5 & 0 & 10 & 10 & 100 & 0 & 120 \\
\hline 6 & 0 & 100 & 10 & 10 & 0 & 120 \\
\hline 7 & 0 & 10 & 10 & 10 & 0 & 120 \\
\hline
\end{tabular}

Table 2 - Modules used in all orders and respective process name and time

\begin{tabular}{|c|c|c|c|c|c|c|c|c|}
\hline Module name & $\mathrm{A}$ & $\mathrm{B}$ & $\mathrm{C}$ & $\mathrm{D}$ & $\mathrm{E}$ & $\mathrm{F}$ & $\mathrm{G}$ & $\mathrm{H}$ \\
\hline Process name & $\mathrm{P}_{1}$ & $\mathrm{P}_{2}$ & $\mathrm{P}_{3}$ & $\mathrm{P}_{4}$ & $\mathrm{P}_{5}$ & $\mathrm{P}_{6}$ & $\mathrm{P}_{7}$ & $\mathrm{P}_{8}$ \\
\hline Process time (seconds) & 1 & 1 & 1 & 5 & 1 & 1 & 1 & 1 \\
\hline
\end{tabular}

Order 1, 2 and 3 require the execution of $\mathrm{PP}_{1}, \mathrm{PP}_{2}$ and $\mathrm{PP}_{3}$ but with different volumes. To guarantee the process execution of these orders, 6 configurations (out of 40320) were found valid: 1, 3471, 10080, 13330, 15472 and 17457 as presented in Table 3 . Since at $\mathrm{t}=0$ only 7 modules are available and there are 8 DPs, one DP will not be assigned any module (N.A.).

Table 3 - Module distribution in DPs for order 1, 2 and 3

\begin{tabular}{|c|c|c|c|c|c|c|c|c|}
\hline Configuration & DP1 & DP2 & DP3 & DP4 & DP5 & DP6 & DP7 & DP8 \\
\hline 1 & A & B & C & N.A. & E & F & G & H \\
\hline 3471 & A & N.A. & B & C & F & E & H & G \\
\hline 10080 & A & N.A. & C & B & E & F & G & H \\
\hline 13330 & A & C & B & N.A. & F & E & H & G \\
\hline 15472 & C & E & A & N.A. & B & G & F & H \\
\hline 17457 & C & N.A. & A & E & B & G & F & H \\
\hline
\end{tabular}

Order 4 requires the execution of $\mathrm{PP}_{1}, \mathrm{PP}_{2}, \mathrm{PP}_{3}$ and $\mathrm{PP}_{5}$. The execution of these PPs simultaneously imposes further constraints on the possible system reconfigurations to perform this order. The possible configurations are depicted in Table 4, and one may observe that only 4 configurations are found valid.

Table 4 - Module distribution in DPs for order 4

\begin{tabular}{|c|c|c|c|c|c|c|c|c|}
\hline Configuration & DP1 & DP2 & DP3 & DP4 & DP5 & DP6 & DP7 & DP8 \\
\hline 1 & A & B & C & N.A. & E & F & G & H \\
\hline 10080 & A & N.A. & C & B & E & F & G & H \\
\hline 15472 & C & E & A & N.A. & B & G & F & H \\
\hline 17457 & C & N.A. & A & E & B & G & F & H \\
\hline
\end{tabular}

At $\mathrm{t}=4$, order 5 is evaluated and it requires a new process $\mathrm{P}_{4}$. Consequently a module that is capable of executing this process needs to be added to the $\mathrm{P} \& \mathrm{P}$ system. It is considered that module $\mathrm{D}$ (capable of performing $\mathrm{P}_{4}$ ) is available and can be plugged in the P\&P system. The processing time of the production module D is 5 seconds. Order 6 and 7 require the same PPs found in order 5 but with different volumes; hence they will share the same possible configurations (see Table 5).

Table 5 - Module distribution in DPs for order 5, 6 and 7

\begin{tabular}{|c|c|c|c|c|c|c|c|c|}
\hline Configuration & DP1 & DP2 & DP3 & DP4 & DP5 & DP6 & DP7 & DP8 \\
\hline 1 & A & B & C & D & E & F & G & H \\
\hline 3250 & A & B & D & C & F & E & H & G \\
\hline 10080 & A & D & C & B & E & F & G & H \\
\hline 10191 & A & C & D & B & F & E & H & G \\
\hline 14769 & C & E & A & B & D & G & F & H \\
\hline 26224 & C & B & A & E & D & G & F & H \\
\hline
\end{tabular}

\section{C) Result Assessment}

The metric scores and results of the simulation are presented in this section. The tests were run 5 times to increase the confidence level and the simulated results had a very small standard deviation (which is justified with the dynamic routing of products based on the previously presented probability function).

The results for order 1, 2 and 3 are depicted in Figure 13 and Figure 14. The analysis of the results shows that a higher score in the MTI metric (which is a consequence of a shorter transport time) does in fact result in the 
improvement of the TP time in the system. In the order 1, the configuration 1 and 10080 (lines are overlapping) have the highest MTI (equal to 0.8) and PSAI (equal to 0.6). That is reflected in a decrease of the average TP time since the products travelled shorter distances and travelled through less non-value adding stations. Similar results can be observed in the order 2 (where the lines of configurations 3471, 13330, 15472 and 17457 are overlapping) and in the order 3 (where the lines of configuration 1 and 10080 are overlapping). Moreover, in the order 3 the higher values of RFI and PDI for configurations 1 and 10080 indicate a bigger routing flexibility and good distribution of products through the transport system respectively.

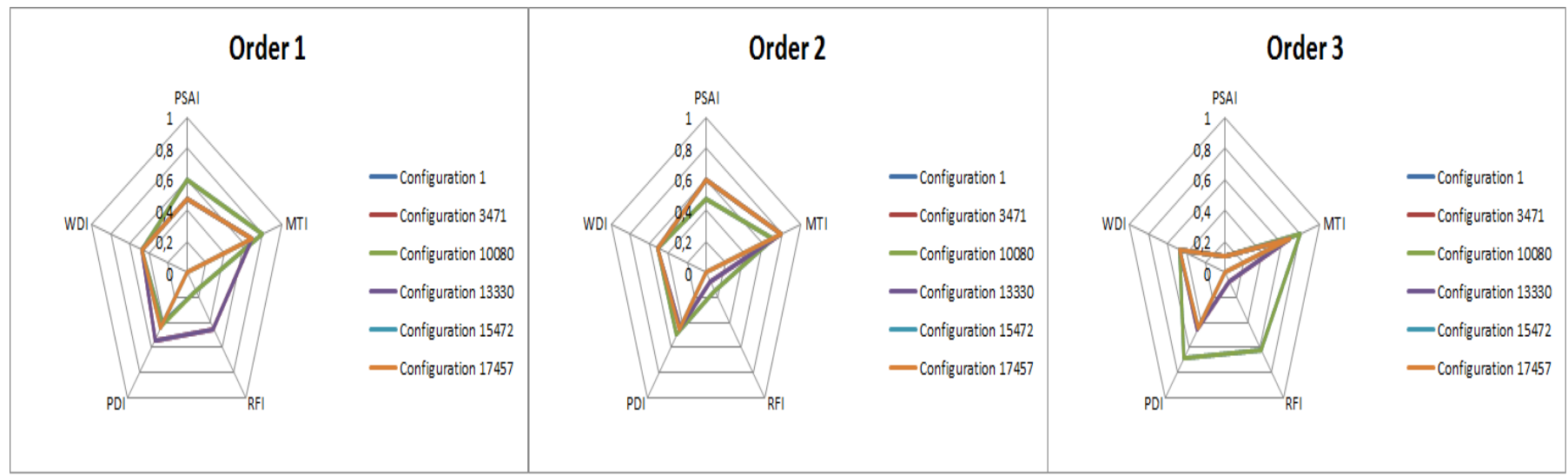

Figure 13 - Configuration scores for orders 1, 2 and 3

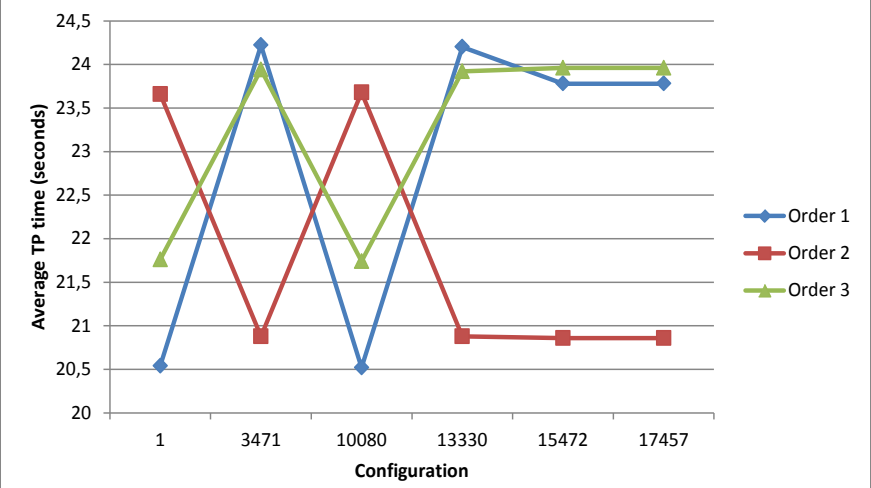

Figure 14 - Average throughput time for orders 1, 2 and 3

The configuration scores and results for order 4 are depicted in Figure 15. The configuration 17457 and 15472 (which lines overlap) have bigger MTI and PSAI indexes and therefore the travel time is reduced. The PDI and RFI values are worse than in configurations 1 and 10080, however, since the system is not overloaded with carriers and the process times are short, following a shortest path becomes more important than distributing products through different routes.

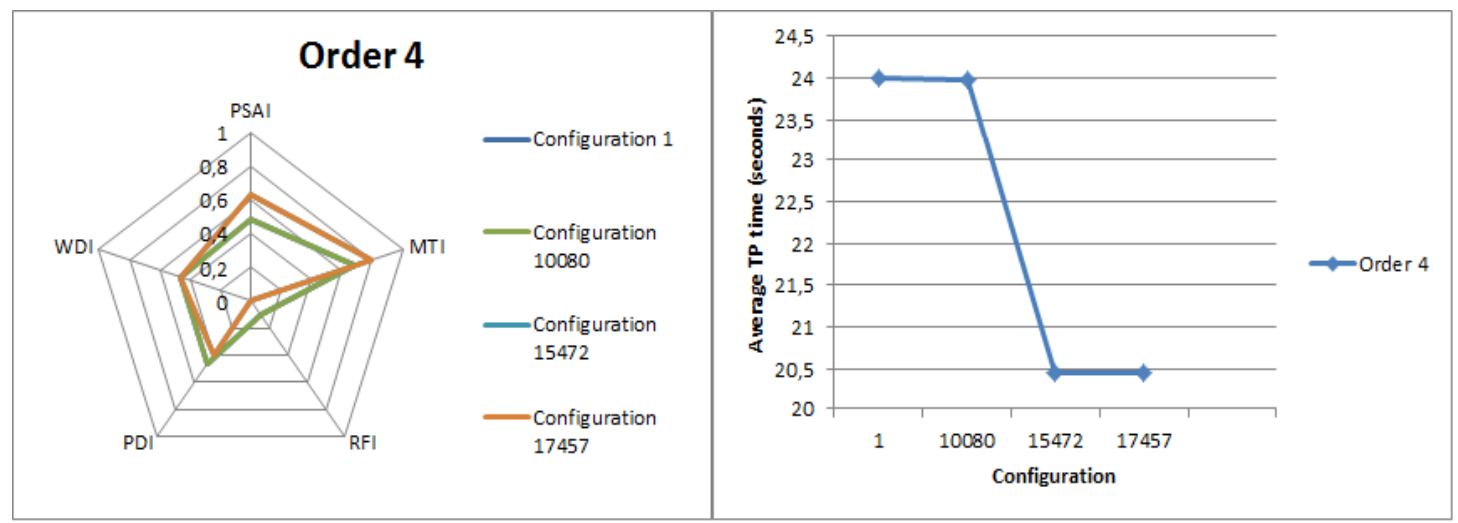

Figure 15 - Configuration scores and average throughput time for order 4 
The results for orders 5, 6 and 7 are depicted in Figure 16 and Figure 17.

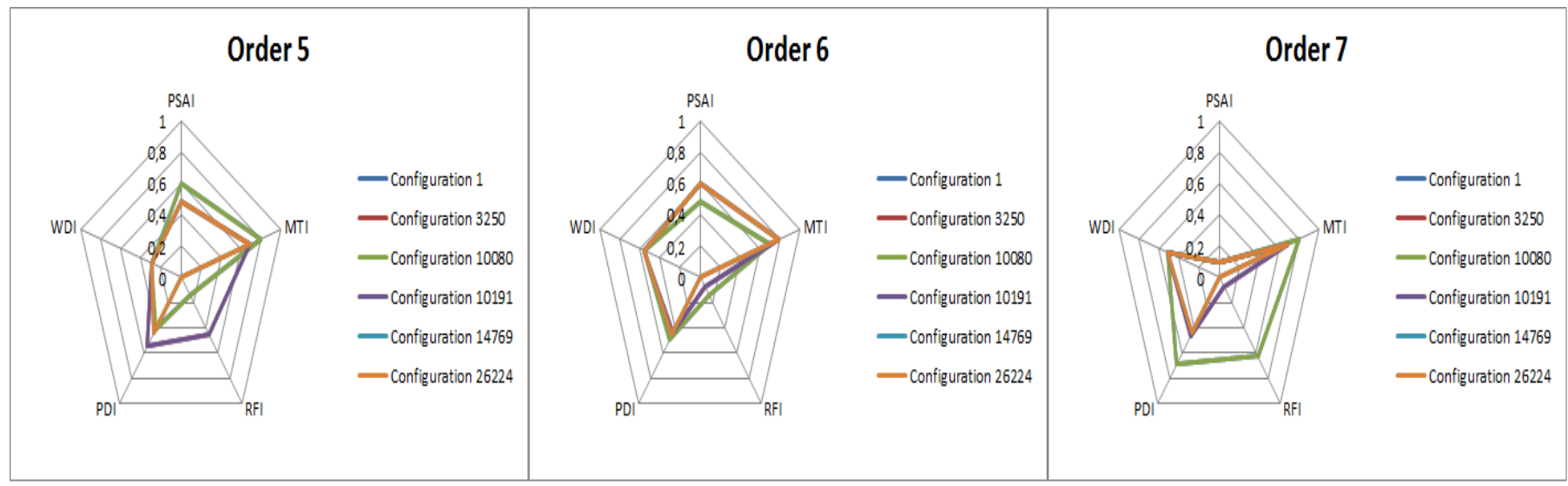

Figure 16 - Configuration scores for orders 5, 6 and 7

The longer processing time of module $\mathrm{D}$ (process $\mathrm{P}_{4}$ ) explains the longer TP time in the results order 5 since 100 products of $\mathrm{PP}_{4}$ are being produced and they require the execution of process $\mathrm{P}_{4}$. Since this process has longer processing time, the TP time gain is lower when considering configurations with more and less travel time and their relation to the MTI and PSAI index. This can be explained by the generation of queues to execute process $\mathrm{P}_{4}$, and in fact one can observe that configurations 1 and 10080 have the highest MTI and PSAI values but lower score on RFI and PDI. This means less routing flexibility and worse distribution of products in the transport system. In order 6 and 7 this is not the case since the volume of $\mathrm{PP}_{4}$ is smaller so the impact on the final average TP time is lower. In these configurations, higher MTI results in better average TP time, i.e. configurations 3250, 10191, 14769 and 26224 in order 6 and configurations 1 and 10080 in order 7.

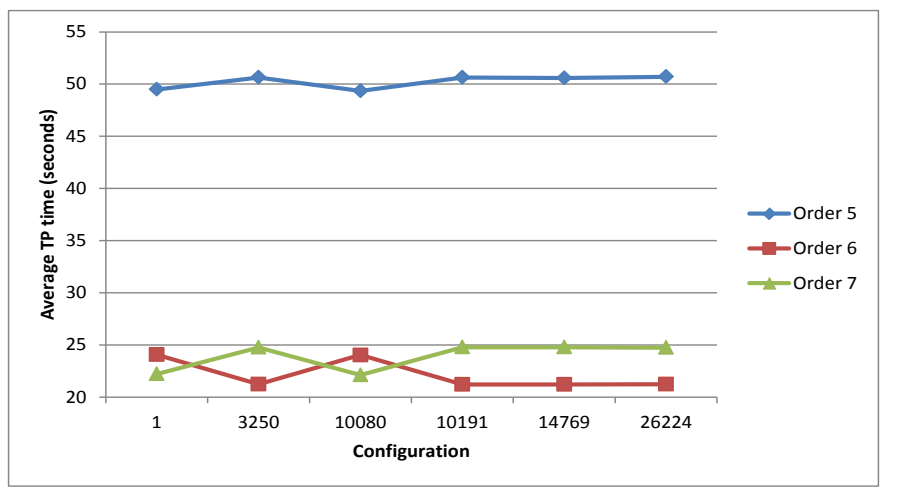

Figure 17 - Average throughput time for orders 5, 6 and 7

The application of the proposed metrics to support reconfiguration selection of P\&P systems include a set of complex decisions to select among the different alternatives. The trade-off solutions that compromise certain objectives in detriment of others will depend on the implications of specific application scenarios. The test-case focused on scenarios where no redundancy of processes exists (1 module for each necessary process) and all modules have similar processing time. Under those assumptions the decision making should be primarily focused on maximizing the MTI and PSAI metrics since those guarantee shorter travelled times and minimisation of non-value adding stations on the production path. Configurations should then be assessed regarding their routing flexibility (RFI) and path distribution (PDI) which can potentially decrease queues and minimize potential impacts of a station failure. The WDI metric is not relevant since the distribution of products in stations will not change due to absence of redundancy. In the case that two or more configurations have exactly the same score in all metrics then the reconfiguration that minimizes the number of module reallocations must be selected. Scenarios with no redundancy of processes but where modules have different processing times require extra attention from the expert user. This is the case of order 5, where a new module that performs process P4 is added to the system and this module has a higher processing time than all other modules. The results obtained in this example suggest that for these cases a new 
metric that estimates the originated queues could be helpful. In any case in the absence of this metric the procedure presented in the case before should be used and special attention should be given to RFI and PDI. According to the aforementioned guidelines the following reconfigurations would be selected: Order 1=1; Order 2=3471; Order 3=10080; Order 4=15472; Order 5=10080; Order 6=3250 and Order 7=1.

Applications with redundancy of processes have not been tested in this article; however they are expected to require a different trade-off between the metrics. In those cases it is expected that the WDI metric will gain expression since it can measure the better distribution of products in the redundant stations. In those cases maximizing the MTI and WDI should be the main priority of the expert user. Next the PSAI should be maximized in order to reduce the number of non-value adding stations and finally the RFI and PDI in order to improve the routing flexibility and distribution of products in the transport paths. Similarly to the previous application scenario, the introduction of a metric that considers the originated queues could be beneficial.

\section{CONCLUSIONS AND FUTURE RESEARCH}

Re-usability will become a fundamental requirement of production as industry needs to decrease resource wastage and footprint. In markets characterized by high customization and dynamicity, approaches that favour re-usability rather than the development of tailored optimized automation systems will pay-off in the long run as their benefits outweigh some of their disadvantages. Nevertheless, to support re-usability of systems and their swift reconfiguration, both a supporting methodology and a decision-making tool are needed.

This article has proposed a method to validate layouts of P\&P systems and reduce the solution space of configuration alternatives through metrics that evaluate the transport and waiting time of different configurations. In the presented test-case the system does not generate large queues due to low process time of stations, period of introduction of new products in the line and asynchronous transport based on individual carriers. For these conditions the MTI metric was found to be the most helpful since it measures the travel time. The PSAI metric can complement this metric by detecting the passage through non-value adding stations which may render a shortest path not the best option. The PDI and WDI give important information about the expected distribution of products in transport paths and stations respectively and therefore they can be used to further narrow the filter between configurations with similar MTI and PSAI for instance. The RFI metric can be used to further narrow down the configurations by selecting the ones with more alternatives. This might be especially valuable in the case where the systems have equipment that is highly prone to failures. The presented results suggest that using the proposed metrics can help filtering under-performing reconfigurations and support the reconfiguration decision in planning activities whenever a new scenario is proposed. Nevertheless, at this stage, they do not substitute entirely production simulation and optimization activities.

The developed tool allows the timely generation and assessment of reconfiguration alternatives that fit the specific production orders that must be produced at each period. This allows the swift reconfiguration of P\&P systems to deal with constantly changing production orders (different product types and volumes) to guarantee the system keeps an acceptable level of performance. One aspect not covered in this research is the possible congestion and queues in the system. In cases where the number of carriers is not well dimensioned or stations do not have similar processing times and generate bottlenecks, then queues might arise in the system. In those cases, shorter travelled distances in specific configurations might not result in a TP time gain.

Future research will encompass further development and testing of the proposed metrics and the consideration of new metrics to estimate queues in the system. To do this, different dynamic routing decisions algorithms and probabilistic estimations will be explored. Furthermore, learning algorithms will be employed to understand the best combination of these metrics and add robustness to the configuration selection decision. Another future research goal is the use of graph theory and complex networks to study different layout structures in order to realize which structures allow better reconfiguration ability. This could be especially useful in the initial process of designing a $\mathrm{P} \& \mathrm{P}$ system to have a structure that allows many reconfigurations and that can therefore cope with constantly changing requirements.

\section{Acknowledgement}

The authors would like to thank the anonymous reviewers for the valuable comments and improvement suggestions. 


\section{REFERENCES}

BABIC, B. 1999. Axiomatic design of flexible manufacturing systems. International Journal of Production Research, 37, 1159-1173.

BABICEANU, R. F. \& SEKER, R. 2015. Manufacturing Cyber-Physical Systems Enabled by Complex Event Processing and Big Data Environments: A Framework for Development. Service Orientation in Holonic and Multi-agent Manufacturing. Springer.

BARATA, J. 2005. "Coalition based approach for shopfloor agility - A multiagent approach", Edições Orion.

BARATA, J., ONORI, M., FREI, R. \& LEITÃO, P. Year. "Evolvable Production Systems: Enabling Research Domains". In: CIRP 2nd International Conference on Changeable, Agile, Reconfigurable, and Virtual Production CARV 2007, 2007 Montreal, Canada.

BECKER, T., MEYER, M. \& WINDT, K. Year. A network theory approach for robustness measurement in dynamic manufacturing systems. In: 17th Annual Cambridge International Manufacturing Symposium, September, Cambridge, 2013.

BECKER, T., MEYER, M. \& WINDT, K. 2014. A manufacturing systems network model for the evaluation of complex manufacturing systems. International Journal of Productivity and Performance Management, 63, 324-340.

BLUNCK, H., VICAN, V., BECKER, T. \& WINDT, K. 2014. Improvement Heuristics for Manufacturing System Design Using Complex Network Figures. Procedia CIRP, 17, 50-55.

CHRYSSOLOURIS, G. 2006. "Manufacturing systems: theory and practice", Springer Verlag.

DIXON, P. M., WEINER, J., MITCHELL-OLDS, T. \& WOODLEY, R. 1987. Bootstrapping the Gini coefficient of inequality. Ecology, 1548-1551.

ELMARAGHY, H., ALGEDDAWY, T., SAMY, S. \& ESPINOZA, V. 2014. A model for assessing the layout structural complexity of manufacturing systems. Journal of Manufacturing Systems, 33, 51-64.

ELMARAGHY, H. A. 2005. Flexible and reconfigurable manufacturing systems paradigms. International Journal of Flexible Manufacturing Systems, 17, 261-276.

FARID, A. M. 2015. Static Resilience of Large Flexible Engineering Systems: Axiomatic Design Model and Measures.

FARID, A. M. \& RIBEIRO, L. Year. An Axiomatic Design of a Multi-Agent Reconfigurable Manufacturing System Architecture. In: 8th International Conference on Axiomatic Design (ICAD 2014), Campus de Caparica, Portugal, 24-26, September 2014, 2014.

GASTWIRTH, J. L. 1972. The estimation of the Lorenz curve and Gini index. The Review of Economics and Statistics, 306-316.

GROOVER, M. P. 2007. Automation, production systems, and computer-integrated manufacturing, Prentice Hall Press.

GROSS, J. L. \& YELLEN, J. 2005. Graph theory and its applications, CRC press.

HU, S. J., KO, J., WEYAND, L., ELMARAGHY, H., LIEN, T., KOREN, Y., BLEY, H., CHRYSSOLOURIS, G., NASR, N. \& SHPITALNI, M. 2011. Assembly system design and operations for product variety. CIRP AnnalsManufacturing Technology, 60, 715-733.

JOHNSON, D. J. 2003. A framework for reducing manufacturing throughput time. Journal of Manufacturing Systems, 22, 283-298.

JOVANE, F., WESTKAMPER, E. \& WILLIAMS, D. 2008. "The ManuFuture Road: Towards Competitive and Sustainable High-Adding-Value Manufacturing", Springer Verlag.

KOREN, Y., HEISEL, U., JOVANE, F., MORIWAKI, T., PRITSCHOW, G., ULSOY, G. \& VAN BRUSSEL, H. 1999. Reconfigurable manufacturing systems. CIRP Annals-Manufacturing Technology, 48, 527-540.

LEITÃO, P., COLOMBO, A. W. \& KARNOUSKOS, S. 2015. Industrial automation based on cyber-physical systems technologies: Prototype implementations and challenges. Computers in Industry.

LEITÃO, P. \& KARNOUSKOS, S. 2015. Industrial Agents: Emerging Applications of Software Agents in Industry, Morgan Kaufmann.

MAFFEI, A. 2012. Characterisation of the Business Models for Innovative, Non-Mature Production Automation Technology. KTH.

MAFFEI, A. \& ONORI, M. 2011. Evolvable Production Systems: Environment for New Business Models. Key Engineering Materials, 467, 1592-1597. 
MCFARLANE, D., GIANNIKAS, V., WONG, A. C. \& HARRISON, M. 2013. Product intelligence in industrial control: Theory and practice. Annual Reviews in Control, 37, 69-88.

MICHALOS, G., MAKRIS, S. \& MOURTZIS, D. 2012. An intelligent search algorithm-based method to derive assembly line design alternatives. International Journal of Computer Integrated Manufacturing, 25, 211-229.

NEGAHBAN, A. \& SMITH, J. S. 2014. Simulation for manufacturing system design and operation: Literature review and analysis. Journal of Manufacturing Systems, 33, 241-261.

NEVES, P., FERREIRA, J., ONORI, M., RIBEIRO, L. \& BARATA, J. Year. Prospection of Methods to Support Design and Configuration of Self-Organizing Mechatronic Systems. In: Systems, Man, and Cybernetics (SMC), 2013 IEEE International Conference on, 2013. IEEE, 3854-3861.

NEVES, P., RIBEIRO, L., DIAS-FERREIRA, J., MAFFEI, A., ONORI, M. \& BARATA, J. Year. Data-mining approach to support layout configuration decision-making in Evolvable Production Systems. In: Systems, Man and Cybernetics (SMC), 2014 IEEE International Conference on, 2014a. IEEE, 3649-3656.

NEVES, P., RIBEIRO, L., DIAS-FERREIRA, J., ONORI, M. \& BARATA, J. Year. Exploring reconfiguration alternatives in self-organising evolvable production systems through simulation. In: Industrial Informatics (INDIN), 2014 12th IEEE International Conference on, 2014b. IEEE, 511-518.

O'MADADHAIN, J., FISHER, D., WHITE, S. \& BOEY, Y. 2003. The jung (java universal network/graph) framework. University of California, Irvine, California.

ONORI, M. \& BARATA, J. 2009. Outlook report on the future of European assembly automation. Assembly Automation, 30, 7-31.

ONORI, M., LOHSE, N., BARATA, J. \& HANISCH, C. 2012. The IDEAS Project: Plug \& Produce at Shop-Floor Level. Assembly Automation, 32, 4-4.

PAPADOPOULOS, H. \& HEAVEY, C. 1996. Queueing theory in manufacturing systems analysis and design: A classification of models for production and transfer lines. European Journal of Operational Research, 92, 127.

PAUKOV, D. 2015. https://code.google.com/p/combinatoricslib/ [Online]. [Accessed February 2nd 2015].

PINE, B. J. 1993. Making mass customization happen: strategies for the new competitive realities. Planning Review, 21, 23-24.

RENZI, C., LEALI, F., CAVAZZUTI, M. \& ANDRISANO, A. 2014. A review on artificial intelligence applications to the optimal design of dedicated and reconfigurable manufacturing systems. The International Journal of Advanced Manufacturing Technology, 72, 403-418.

RIBEIRO, L., BARATA, J., COLOMBO, A. \& SELIGENSTADT, G. 2008. MAS and SOA: A Case Study Exploring Principles and Technologies to Support Self-Properties in Assembly Systems. SARC'2008: Self-Adaptation for Robustness and Cooperation in Holonic Multi-Agent Systems, 43.

RIBEIRO, L., ROSA, R., CAVALCANTE, A. \& BARATA, J. Year. IADE - IDEAS Agent Development Environment: Lessons Learned and Research Directions. In: CIRP Conference On Assembly Technologies And Systems, 2012 Michigan, USA. IEEE, 2752-2757.

SHEN, W., HAO, Q., YOON, H. J. \& NORRIE, D. H. 2006. Applications of agent-based systems in intelligent manufacturing: An updated review. Advanced Engineering Informatics, 20, 415-431.

SHIYAS, C. \& PILLAI, V. M. 2014. A mathematical programming model for manufacturing cell formation to develop multiple configurations. Journal of Manufacturing Systems, 33, 149-158.

UEDA, K. Year. "A concept for bionic manufacturing systems based on DNA-type information". In: 8th International PROLAMAT Conference on Human Aspects in Computer Integrated Manufacturing, 1992. North-Holland Publishing Co., 863.

VALCKENAERS, P., VAN BRUSSEL, H., BONGAERTS, L. \& WYNS, J. 1997. Holonic manufacturing systems. Integrated Computer-Aided Engineering, 4, 191-201.

WEBBINK, R. F. \& HU, S. J. 2005. Automated generation of assembly system-design solutions. Automation Science and Engineering, IEEE Transactions on, 2, 32-39.

WIENDAHL, H. P., ELMARAGHY, H. A., NYHUIS, P., ZÄH, M. F., WIENDAHL, H. H., DUFFIE, N. \& BRIEKE, M. 2007. Changeable manufacturing-classification, design and operation. CIRP Annals-Manufacturing Technology, 56, 783-809. 
WILLIAMS, D., RATCHEV, S., CHANDRA, A. \& HIRANI, H. 2006. The application of assembly and automation technologies to healthcare products. CIRP Annals-Manufacturing Technology, 55, 617-642.

WONG, C. Y., MCFARLANE, D. \& AGARWAL, V. Year. The intelligent product driven supply chain. In: Systems, Man and Cybernetics, 2002 IEEE International Conference On, 2002. IEEE, 6 pp. vol. 4.

YU, J.-C. \& LI, Y.-M. 2006. Structure representation for concurrent analysis of product assembly and disassembly. Expert systems with Applications, 31, 705-714. 